\title{
Prediction of Response to Erythropoiesis Stimulating Agents in Low-Risk Myelodysplastic Syndromes
}

\author{
Saroj Hattakitpanitchakul ${ }^{1}$, Sirorat Kobbuaklee ${ }^{1,2}$, Kitsada Wudhikarn ${ }^{1,2}$, \\ Chantana Polprasert ${ }^{1,2 *}$
}

\begin{abstract}
Introduction: Erythropoiesis stimulating agents (ESAs) represents the principal treatments for anemia in patients with lower-risk myelodysplastic syndromes (MDS). Pre-treatment erythropoietin (EPO) level and previous blood transfusion requirement are the two major predictors for response to ESAs. However, most evidence was derived from Western countries whereas there have been limited data in patients with Asian background. Methods: We retrospectively collected data on patients with low-risk MDS who received ESAs. Erythroid response was evaluated according to IWG 2006 criteria. MDS subtypes, r-IPSS, baseline hemoglobin (Hb), ESAs dosage and erythropoietin level were reviewed from medical records. Gene mutations were analyzed in patients' blood or bone marrow at diagnosis by 40 -gene myeloid panel targeted sequencing. Clinical and laboratory parameters were compared between erythroid responder and non-responder groups. Results: A total of 47 patients were recruited in the study. The median age at diagnosis of the patients in this cohort was 77 years (IQR, 70-83) and $44.7 \%$ were male. The median revised international prognostic scoring system (R-IPSS) score of patients was 2.5. Response rate to ESAs was 46.8\% (22/47). Median EPO level in responders was significantly lower than non-responders (27.7 vs. $59.1 \mathrm{U} / \mathrm{L}, \mathrm{p}=0.02)$. Median ESAs dosage in responder group was 30,000 units per week. Cytogenetic abnormalities were detected in $27.3 \%$ and $24 \%$ of the responder and non-responder groups, respectively. Of 22 patients with available 40 gene mutation targeted sequencing, ASXL1, IDH2 and TET2 represented the 3 most common mutations and were found in $22 \%, 22 \%$ and $17 \%$, respectively. There were no differences in cytogenetic abnormalities and gene mutations between groups. Patients who responded to ESAs showed a higher 5-year overall survival (OS) compared to non-responders (5-year OS 75\% vs. $60.9 \% ; \mathrm{p}=0.008$ ). Conclusion:We conclude that a low serum $\mathrm{EPO}$ level is a predictive factor for responsiveness to ESAs in Asian patients with low-risk MDS.
\end{abstract}

Keywords: Low-risk myelodysplastic syndromes- erythropoiesis stimulating agents- erythropoietin level- gene mutations

Asian Pac J Cancer Prev, 22 (12), 4037-4042

\section{Introduction}

Myelodysplastic syndromes (MDS) are clonal myeloid disorders characterized by morphological dysplasia and ineffective hematopoiesis leading to peripheral blood cytopenia and increased risk of progression to acute myeloid leukemia (AML) (Steensma and Bennett, 2006; Hellstorm-Lindberg et al., 1997). The natural history and prognosis of MDS are classified by the International Prognostic Scoring System (IPSS) and Revised International Prognostic Scoring System (R-IPSS). In low-risk MDS (low or intermediate-1 IPSS or R-IPSS $\leq 3.5$ ), anemia is the predominant feature, often leading to red blood cell (RBC) transfusion requirement, poor quality of life, and worsening cardiovascular comorbidities (Steensma and Bennett, 2006). Correcting anemia by transfusion support or enhancing intrinsic hematopoiesis is the central approach of treatment for patients with lower risk MDS with the goal hemoglobin $(\mathrm{Hb})$ of $10 \mathrm{~g} / \mathrm{dL}$. Erythropoiesis stimulating agents (ESAs) has been the major treatment modality for lower risk MDS aiming to alleviate symptoms, reduce transfusion requirement and improve quality of life in these patients (Newman et al., 2012). Previous studies showed that patients with a history of frequent blood transfusions and high EPO levels are less likely to respond to ESAs (Hellstorm-Lindberg et al., 1997).

The National Comprehensive Cancer Network (NCCN) guideline 2021, version 3, recommends starting ESAs in low-risk MDS patients with an EPO level below $500 \mathrm{U} / \mathrm{L}$ with a dosage of 40,000-60,000 U/week (Greenberg et al., 2021). The Nordic MDS group recommended ESA dosage starting at 30,000 U/week (Kjeldsen et al., 2014). Based on the above guidelines, the Thai MDS study group

${ }^{1}$ Department of Medicine, Faculty of Medicine, Chulalongkorn University, King Chulalongkorn Memorial Hospital, Bangkok, Thailand. ${ }^{2}$ Research Unit in Translational Hematology, Chulalongkorn University, Bangkok, Thailand. *For Correspondence: jeedchantana@gmail.com 
also suggested to initiate treatment with ESAs in low-risk MDS with an EPO level $<500 \mathrm{U} / \mathrm{L}$ at a starting dose of 30,000-40,000 U/week (Hellstorm-Lindberg et al., 1997; Suporn et al., 2011). These recommendations were based on studies of Western populations. Herein, we conducted this study, focusing on MDS patients in Thailand to validate whether effective dosage levels derived from Western studies can be generalized to Asian populations or whether dosage modifications are necessary.

We aimed to examine EPO level as a predictor of ESAs responsiveness and to identify optimal dosage of ESAs treatment. In addition to EPO level, other relevant clinical factors and mutational attributes were also analyzed to determine potential association with ESAs responsiveness.

\section{Materials and Methods}

\section{Patients and study design}

We retrospectively reviewed medical records of low-risk MDS patients who received ESAs at King Chulalongkorn Memorial Hospital between January 2014 and March 2020. MDS was diagnosed by bone marrow aspiration and biopsy according to WHO classification 2016 (Arber et al., 2016). Patients were categorized using the R-IPSS into a low-risk group (R-IPSS $\leq 3.5$ ), and a high-risk group (R-IPSS > 3.5) (Santini 2011). Inclusion criteria for this study were defined as: 1) Low-risk MDS (R-IPSS score $\leq 3.5$ ), 2) Age $\geq 18$ years old, and 3) Patients received ESA treatment. Patients with therapy-related MDS and history of pure red cell aplasia were excluded. Erythroid response was evaluated at 4 and 8 weeks after treatment according to International Working Group criteria (IWGc) (Cheson et al., 2006).

We abstracted relevant clinical information including baseline characteristics, comorbidities, R-IPSS score, MDS subtype, EPO level, gene mutations, chromosome study, reticulocyte count, complete blood count at baseline, 4 weeks, and 8 weeks after the treatment with ESAs, and dosage of ESAs from electronic medical records. According to the erythroid response criteria, patients were classified into two groups (responder and non-responder).

Erythroid response criteria were defined as: 1) Hb level increases $\geq 1.5 \mathrm{~g} / \mathrm{dL}$, or 2 ) relevant reduction of units of $\mathrm{RBC}$ transfusion by an absolute number of at least $4 \mathrm{RBC}$ transfusions/ 8 weeks compared with the pretreatment transfusion numbers in the previous 8 weeks. Only RBC transfusions given for a $\mathrm{Hb}$ level $\leq 9.0 \mathrm{~g} / \mathrm{dL}$ at pretreatment were counted in the RBC transfusion response evaluation (IWGc) Cheson et al., 2006). This study was approved by the Institutional Review Board, Faculty of Medicine, Chulalongkorn University, Bangkok, Thailand (IRB number 450/62).

For targeted sequencing, DNA from bone marrow or peripheral blood was extracted using Gentra Puregene Blood Kit (Qiagen). Samples were processed and sequenced with IONS5 sequencer using custom 40-gene myeloid panel (Supplementary Table 1). Extracted DNA were prepared using the Ion ChefTM system. Variants were analyzed using Ion ReporterTM software as previously described (Christina Ferrone et al., 2018).
Serum erythropoietin level was measured using chemiluminescence technique using IMMULITE 2000 system-erythropoietin (EPO) Assay.

\section{Statistical analysis}

Continuous variables were described using median and interquartile range using student's t test or Wilcoxon rank sum test for comparison between groups. Categorical parameters were report as frequency and percentage. The comparison of categorical data between two groups were performed using Chi-square or Fisher exact test. Patient characteristics (comorbidities, R-IPSS, subclasses of MDS, EPO level, gene mutations, chromosome study, reticulocyte count) from each of the two groups were compared by Chi square test and Wilcoxon rank sum test. ESAs dosage in the responder group was demonstrated by median value. Survival analysis was performed using Kaplan-Meier estimate with log-rank comparison. Univariate logistic regression analysis was performed to determine predictive factor for ESA responsiveness. P-value less than 0.05 were considered statistically significance. All statistical analyses were performed using Stata version 15.1 .

\section{Results}

A total of 47 low-risk MDS patients were included in this study. Baseline patient characteristics are displayed in Table 1. Gender and age did not show differences between responder and non-responder groups. MDS with multilineage dysplasia (MLD) was the most common subtype $(59.6 \%)$, followed by single lineage dysplasia (SLD) $36.2 \%$, MDS with ring sideroblast (RS) $2.1 \%$, and excess blast-1 (EB-1) 2.1\%. Erythroid response was evaluated at 4 and 8 weeks after ESA treatment. Of 47 patients, response to ESA was observed in 22 patients $(46.8 \%)$. There was no difference in median R-IPSS score and cytogenetic abnormalities between responder and non-responder groups. Subtype of MDS was not different between the 2 groups. Median R-IPSS score was 2.5 , which was similar in both groups. Cytogenetic abnormalities were detected in $27.3 \%$ and $24.0 \%$ of the responder and non-responder groups, respectively $(\mathrm{p}=0.79)$ (Supplementary Table 2$)$.

The median EPO levels were 59.1(IQR 25.2-185) U/L and 27.7(IQR 13.1-58.5) U/L in the non-responder and responder groups, respectively $(\mathrm{p}=0.02)$ (shown in Table 2 and Fig. 1). Hb levels at baseline were not significantly different between the responder and non-responder groups ( 8.5 vs $8 \mathrm{~g} / \mathrm{dL}, \mathrm{p}=0.52$ ). After treatment with ESAs, $\mathrm{Hb}$ levels in the responder group were significantly higher than those in the non-responder group at 4 weeks ( $10.3 \mathrm{vs.}$ $7.7 \mathrm{~g} / \mathrm{dL}, \mathrm{p}<0.001)$ and 8 weeks (10.9 vs. 8.1, $\mathrm{p}<0.001)$, respectively (shown in Table 3 ). In the erythroid responder group, median dose of ESA was 30,000 units per week (10,000-60,000). The median duration of response in responder group is 24 months (IQR 10-28 months).

Next generation sequencing using 40-gene myeloid panel was completed for 22 cases (11 responders, 11 non-responders). The most frequent gene mutations were ASXL1 (22\%), IDH2 (22\%) and TET2 (17\%), 


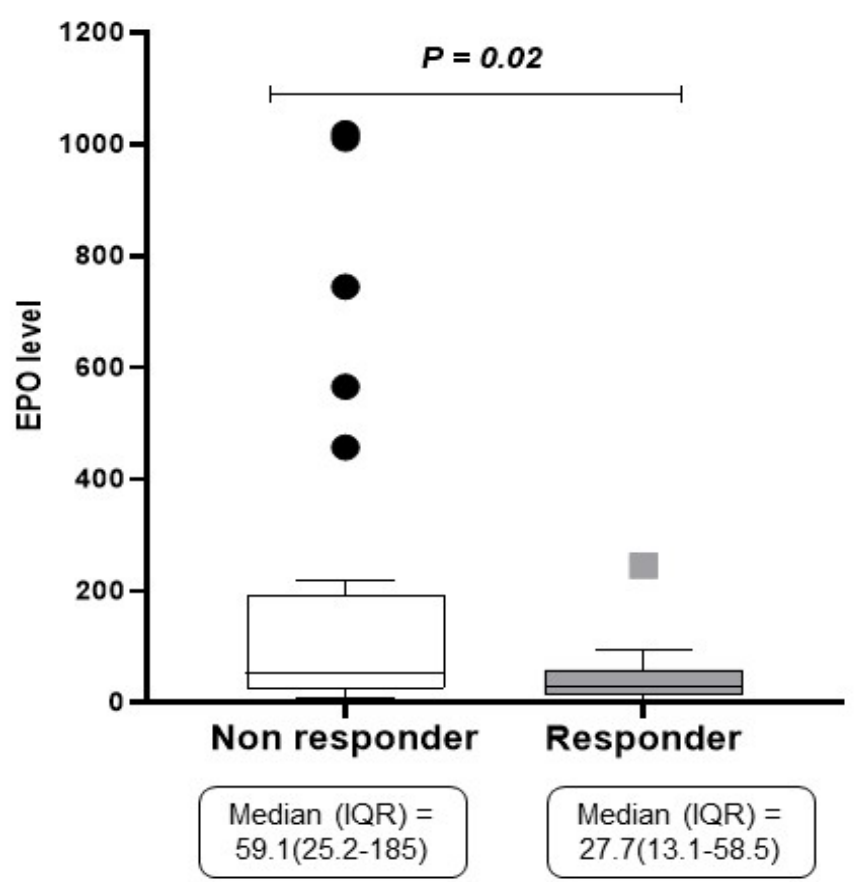

EPO: Erythropoietin, ESA: Erythropoietin stimulating agent

Figure 1. Comparing Erythropoietin (EPO) Level between Erythropoiesis Stimulating Agents (ESAs) Response Group Using Wilcoxon Rank Sum Test.

respectively. There were no differences in gene mutations found between the 2 groups (shown in Figure 2 and Supplementary Table 3 and 4). Patients who responded to ESAs showed better 5-year OS rate compared to non-responders $(75 \%$ vs. $60.9 \% ; \mathrm{p}=0.008)$ (shown in Figure 3).

\section{Discussion}

In line with the data from the western countries (Santini 2011; Wallvik et al., 2002). EPO level in the responder group is significantly lower than in the non-responder group. However, the level of EPO in non-responder group in our study is much lower than what was reported in the

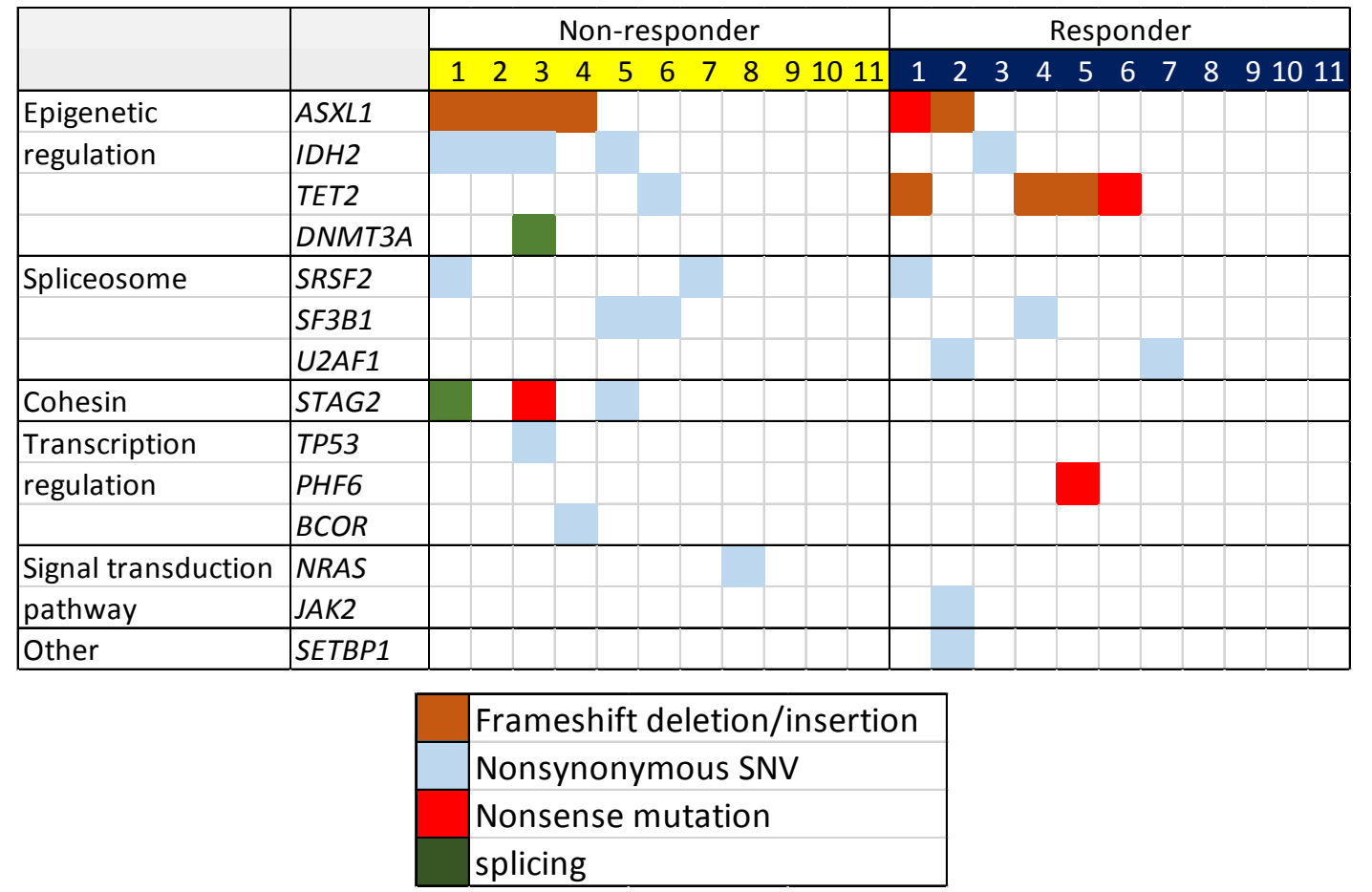

Figure 2. Gene Mutations in Responder and Non-Responder Groups 
Table 1. Characteristics of the Patients

\begin{tabular}{|c|c|c|c|c|}
\hline & $\begin{array}{c}\text { Total } \\
(\mathrm{N}=47)\end{array}$ & $\begin{array}{l}\text { No response } \\
(\mathrm{N}=25)\end{array}$ & $\begin{array}{c}\text { Response } \\
(\mathrm{N}=22)\end{array}$ & $\begin{array}{c}\text { P- } \\
\text { value }\end{array}$ \\
\hline Sex, $\mathrm{n}(\%)$ & & & & $0.49^{\mathrm{a}}$ \\
\hline Male & $21(44.7)$ & $10(40)$ & $11(50)$ & \\
\hline Female & $26(55.3)$ & $15(60)$ & $11(50)$ & \\
\hline Subclass, n (\%) & & & & $0.56^{\mathrm{a}}$ \\
\hline MLD & $27(57.5)$ & $15(60)$ & $12(54.5)$ & \\
\hline SLD & $18(38.3)$ & $9(36)$ & $9(41)$ & \\
\hline RS-SLD & $1(2.1)$ & $1(4)$ & $0(0)$ & \\
\hline EB1 & $1(2.1)$ & $0(0)$ & $1(4.5)$ & \\
\hline \multicolumn{5}{|l|}{ Underlying, $\mathrm{n}(\%)$} \\
\hline DM & $11(23.4)$ & $8(32)$ & $3(13.6)$ & $0.14^{\mathrm{a}}$ \\
\hline Hypertension & $20(42.6)$ & $12(48)$ & $8(36.4)$ & $0.42^{\mathrm{a}}$ \\
\hline Renal & $5(10.6)$ & $1(4)$ & $4(18.2)$ & $0.17^{\mathrm{a}}$ \\
\hline Cytogenetic & & & & $0.79^{\mathrm{a}}$ \\
\hline Normal & $35(74.5)$ & $19(76)$ & $16(72.7)$ & \\
\hline Abnormal & $12(25.5)$ & $6(24)$ & $6(27.3)$ & \\
\hline RIPSS , median (p25-p75) & $2.5(2-2.5)$ & $2.5(2-2.5)$ & $2.5(2-2.5)$ & $0.67^{\mathrm{b}}$ \\
\hline BUN, median (p25-p75) & $18(13-26)$ & $17(13-25)$ & $18(13-31)$ & $0.76^{\mathrm{b}}$ \\
\hline Creatinine, median (p25-p75) & $1.07(0.78-1.24)$ & $1.02(0.74-1.16)$ & $1.13(0.85-1.29)$ & $0.23^{\mathrm{b}}$ \\
\hline Reticulocyte count, median (p25-p75) & $52000(33000-88000)$ & $44000(28000-82000)$ & $65000(39000-89000)$ & $0.35^{\mathrm{b}}$ \\
\hline Ferritin, median (p25-p75) & $808(318-1304)$ & $820(325-1157)$ & $771(239-1773)$ & $0.53^{\mathrm{b}}$ \\
\hline Blood transfusion per month, median (p25-p75) & $0(0-0)$ & $0(0-5)$ & $0(0-0)$ & $0.13^{b}$ \\
\hline \multicolumn{5}{|l|}{$\mathrm{CBC}$} \\
\hline Hemoglobin (g/dL) & $8.3(7.3-8.9)$ & $8(7.4-8.8)$ & $8.5(7.3-9)$ & 0.52 \\
\hline Hematocrit $(\%)$ & $26.1(23-28.1)$ & $25.7(22.7-28.1)$ & $26.8(23.4-28.2)$ & 0.41 \\
\hline $\mathrm{WBC}\left(\mathrm{Cell} / \mathrm{mm}^{3}\right)$ & $4820(3500-5710)$ & $4555(3135-5425)$ & $5090(4190-6540)$ & 0.18 \\
\hline Neutrophil (\%) & $57(48-66.2)$ & $54.3(39.7-64.2)$ & $58.1(53.7-68.9)$ & 0.12 \\
\hline Platelet $\left(\times 10^{3} / \mathrm{mm}^{3}\right)$ & $166(97-262)$ & $142(92-263)$ & $170(101-262)$ & 0.65 \\
\hline
\end{tabular}

${ }^{a}$ P-value from chi-square test; ${ }^{b}$ P-value from Wilcoxon rank sum test; MLD, Multilineage dysplasia; SLD, Single lineage dysplasia; RS-SLD, Ring sideroblast with single lineage dysplasia; EB1, Excess blast-1; DM, Diabetes mellitus; R-IPSS, Revised international prognostic score system; BUN, Blood urea nitrogen; $\mathrm{p} 25-\mathrm{p} 7,: 25^{\text {th }}$ percentile $-75^{\text {th }}$ percentile; WBC, White blood cell

Caucasian population. Possible causes of different EPO levels among the studies could be due to differences in patient or clinical characteristics such as ethnicity, baseline R-IPSS, and different subtype of MDS. The maximum EPO level in the responder group of our study was 245 $\mathrm{U} / \mathrm{L}$, suggesting that erythropoietin cut-off levels suitable for ESAs treatment for Asian patients might be lower than Western patients. Since ESAs usage has been reported to be associated with a 1.6-fold increased risk of myocardial infarction and thrombotic complications, conservative use of ESAs should be maintained to prevent adverse events (Lippi et al., 2010; Horvath-Puho et al., 2018). However, the appropriated cut-off EPO level should be further studied in a larger cohort.

Using ITACA score (Buckstein et al., 2017) and the Nordic group's score (Hellstorm-Lindberg et al., 2003) in our cohort showed lower response rates compared to the original studies (Supplementary Table 5 and 6). Predictive scoring model for Asian patients should be considered for more accurate prediction of ESA responsiveness.

The median ESA dosage in the responder group of $30,000 \mathrm{U} /$ week suggests that most of the Thai low-risk MDS patients required the lowest recommended dosage of ESAs according to the guidelines (Greenberg et al., 2021). Overall, hemoglobin level pre-treatment in our cohort was 7.3-8.9 g/dL. We demonstrated overall survival benefit in patients who responded to ESAs which is concordant with the results of Messa et al., (2019) who reported an improvement in survival of patients with erythroid response with hemoglobin 8-10 g/dL pre-treatment. Limitations of this study are the small number of patients and the unavailability of DNA for target sequencing in all

Table 2. Comparison of Erythropoietin (EPO) Level between Non-Responder and Responder Group

\begin{tabular}{lcccc}
\hline & Total $(\mathrm{N}=47)$ & Non responder $(\mathrm{N}=25)$ & Responder $(\mathrm{N}=22)$ & P-value \\
\hline EPO level, Mean (IQR) & $37.4(15.7-91.5)$ & $59.1(25.2-185)$ & $27.7(13.1-58.5)$ & 0.02 \\
\hline P-value from Wilcoxon rank sum test; EPO, Erythropoietin
\end{tabular}

Pilcoxon rank sum test; EPO, Erythropoietin 
Table 3. Comparison of CBC between Non-Responder Group and Responder Group

\begin{tabular}{|c|c|c|c|c|}
\hline & Total $(\mathrm{N}=47)$ & Non responder $(\mathrm{N}=25)$ & Responder $(\mathrm{N}=22)$ & P-value \\
\hline \multicolumn{5}{|c|}{ Hemoglobin $(\mathrm{g} / \mathrm{dL})$} \\
\hline Week 0 & $8.3(7.3-8.9)$ & $8(7.4-8.8)$ & $8.5(7.3-9)$ & 0.52 ; Ref \\
\hline Week 4 & $9.1(7.4-10.4)$ & $7.7(6.2-9.3)$ & $10.3(8.9-11.6)$ & $<0.001$ \\
\hline Week 8 & $9.4(7.7-10.9)$ & $8.1(6.9-9.4)$ & $10.9(10.3-12.7)$ & $<0.001$ \\
\hline \multicolumn{5}{|c|}{ Hematocrit (\%) } \\
\hline Week 0 & $26.1(23-28.1)$ & $25.7(22.7-28.1)$ & $26.8(23.4-28.2)$ & 0.41 ; Ref \\
\hline Week 4 & $28(22.6-32.8)$ & $23.1(18.8-29.8)$ & $31.6(28-37.1)$ & $<0.001$ \\
\hline Week 8 & $29.9(24.8-33.1)$ & $26.2(22.9-29.4)$ & $33.4(32.4-40.6)$ & $<0.001$ \\
\hline \multicolumn{5}{|c|}{ WBC (Cell/mm3) } \\
\hline Week 0 & $4820(3500-5710)$ & $4555(3135-5425)$ & $5090(4190-6540)$ & 0.18 ; Ref \\
\hline Week 4 & $4800(3280-5890)$ & $4280(2740-6010)$ & $5250(3889-5770)$ & 0.93 \\
\hline Week 8 & $4105(2820-7310)$ & $3950(2675-6205)$ & 4445 (3465-8195) & 0.54 \\
\hline \multicolumn{5}{|c|}{ Neutrophil (\%) } \\
\hline Week 0 & $57(48-66.2)$ & $54.3(39.7-64.2)$ & $58.1(53.7-68.9)$ & 0.12 ; Ref \\
\hline Week 4 & $57.4(38.3-64.5)$ & $46(34.4-63.1)$ & $60.3(48.8-72.6)$ & 0.17 \\
\hline Week 8 & $57.5(43.9-66.5)$ & $50.6(38.6-61)$ & $63(51.7-70.3)$ & 0.04 \\
\hline \multicolumn{5}{|c|}{ Platelet (x103/mm3) } \\
\hline Week 0 & $166(97-262)$ & $142(92-263)$ & $170(101-262)$ & 0.65 ; Ref \\
\hline Week 4 & $200(88-274)$ & $213(84-354)$ & $155(101-242)$ & 0.11 \\
\hline Week 8 & $141(80-284)$ & $115(74-264)$ & $154(106-337.5)$ & 0.86 \\
\hline
\end{tabular}

Data present Median ( $25^{\text {th }}$ percentile- $75^{\text {th }}$ percentile), P-value from Generalized estimating equations (GEE) with linear model; WBC, White blood cell

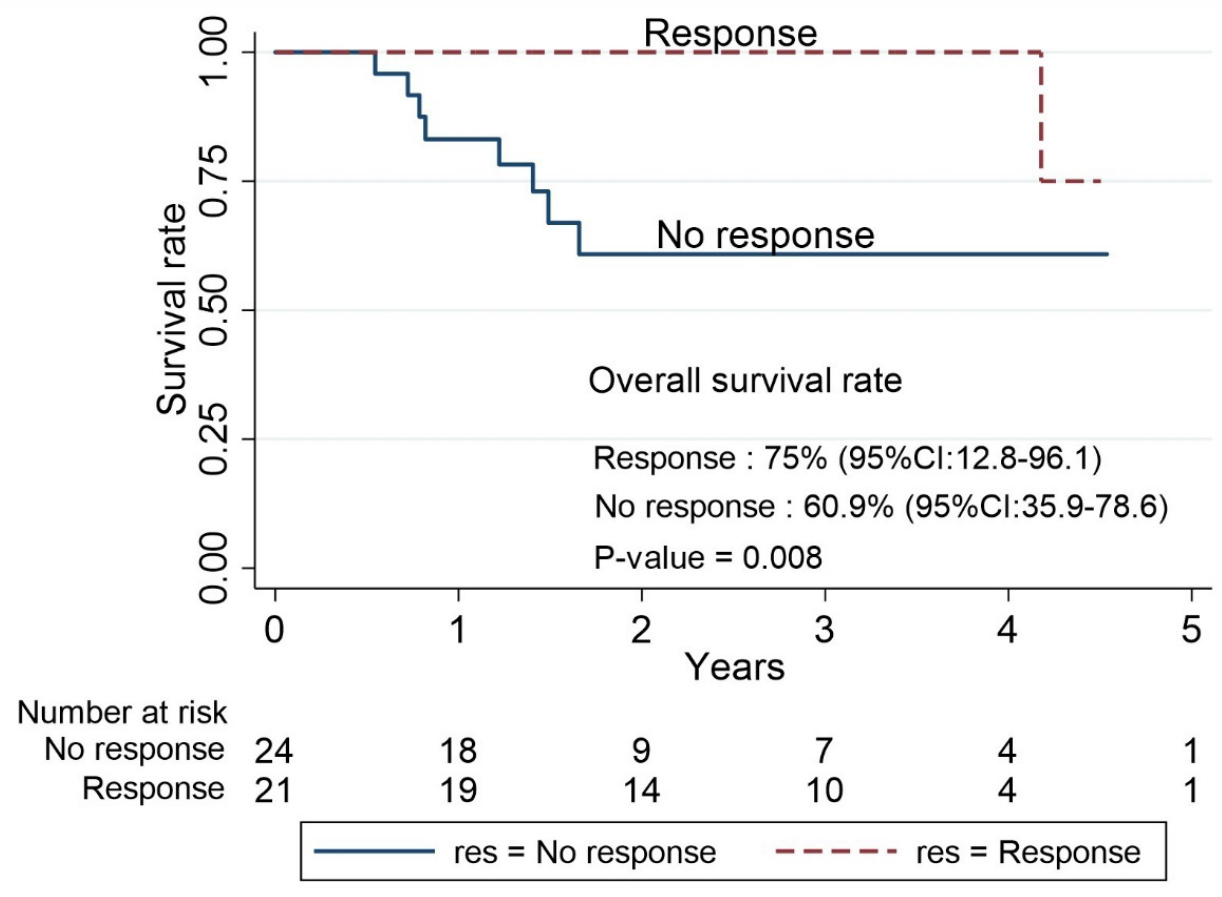

Figure 3. Overall Survival Rate in Responder and Non-Responder Groups

patients. However, this is one of the few studies in Asia that incorporated NGS data into clinical information. Further studies with a larger number of patients from Asia are recommended. Administering reduced dosages, i.e., $20,000 \mathrm{U}$ per week or more with the spread-out schedule i.e., 30,000 U per every 10 days in Asian population, would be an interesting issue to be investigated.
In conclusions, results of this study showed that low serum erythropoietin (EPO) levels were associated with erythroid response to ESAs. EPO levels in the Thai MDS were lower than those of the Western MDS. Patients who achieved erythroid response from ESAs treatment showed better survival benefit than the non-responders. 


\section{Author Contribution Statement}

S.H. collected clinical data, analyzed, and wrote manuscript. S.K. extracted DNA and performed sequencing experiments. K.W. provided clinical data, conceptualized, and wrote manuscript. C.P. collected clinical data, analyzed, wrote, and conceptualized the manuscript.

\section{Acknowledgements}

Authors thank Professor Chongrak Polprasert and the research team of the Department of Medicine, Faculty of Medicine, Chulalongkorn University for editing the final manuscript.

\section{Funding statement}

This research was supported bya Ratchadapiseksompotch Fund, Faculty of Medicine, Chulalongkorn University, grant number RA63/087 (S.H. and C.P.), a grant from Thailand Research Fund, grant number RDG6050109 (C.P.), and a grant from Research Unit in Translational Hematology (K.W.).

\section{Ethics approval}

The institutional review board of the Faculty of Medicine, Chulalongkorn University approved the study (IRB number 450/62). All patients provided written informed consent granting investigators permission to abstract their medical information for research purposes.

\section{Availability of data}

All data generated or analyzed during this study are included in this article and its supplementary material files. Further enquiries can be directed to the corresponding author.

\section{Conflict of interest}

The authors declared no potential conflicts of interest.

\section{References}

Arber DA, Orazi A, Hasserjian R, et al (2016). The 2016 revision to the World Health Organization classification of myeloid neoplasms and acute leukemia. Blood, 127, 2391-405.

Buckstein R, Balleari E, Wells R, et al (2017). ITACA: A new validated international erythropoietic stimulating agentresponse score that further refines the predictive power of previous scoring systems. Am J Hematol, 92, 1037-46.

Cheson BD, Greenberg PL, Bennett JM, et al (2006). Clinical application and proposal for modification of the International Working Group (IWG) response criteria in myelodysplasia. Blood, 108, 419-25.

Christina Ferrone HW, Laura S, Brooke S, et al (2018). Validation and clinical impact of the oncomine myeloid targeted DNA and RNA ion semiconductor sequencing assay. Blood, 132, 5523.

Greenberg PL, Stone RM, Al-Kali A, et al (2021). Myelodysplastic Syndromes, Version 3.2021. Clin Pract Guidelines Oncol, 15.

Hellstrom-Lindberg E, Negrin R, Stein R, et al (1997). Erythroid response to treatment with G-CSF plus erythropoietin for the anaemia of patients with myelodysplastic syndromes: proposal for a predictive model. Br J Haematol, 99, 344-51.

Hellstrom-Lindberg E, Gulbrandsen N, Lindberg G, et al (2003). A validated decision model for treating the anaemia of myelodysplastic syndromes with erythropoietin+granulocyte colony-stimulating factor: significant effects on quality of life. Br J Haematol, 120, 1037-46.

Horvath-Puho E, Suttorp MM, Frederiksen H, et al (2018). Erythropoiesis-stimulating agents and cardiovascular events in patients with myelodysplastic syndrome and multiple myeloma. Clin Epidemiol, 10, 1371-80.

Kjeldsen L, Dybedal I, Hellström-Lindberg E, et al (2014). Guidelines for the diagnosis and treatment of Myelodysplastic Syndromes and Chronic Myelomonocytic Leukemia, Nordic MDS Group. 2014.

Lippi G, Franchini M, Favaloro EJ (2010). Thrombotic complications of erythropoiesis-stimulating agents. Semin Thromb Hemost, 36, 537-49.

Messa E, Gioia D, Masiera E, et al (2019). Effects of erythropoiesis-stimulating agents on overall survival of International Prognostic Scoring System Low/Intermediate-1 risk, transfusion-independent myelodysplastic syndrome patients: a cohort study. Haematologica, 104, 4-8.

Newman K, Maness-Harris L, El-Hamaidi I, et al (2012). Revisiting use of growth factors in myelodysplastic syndromes. Asian Pac J Cancer Prev, 13, 1081-91.

Santini V (2011). Clinical use of erythropoietic stimulating agents in myelodysplastic syndromes. Oncologist, 16, 35-42.

Steensma DP, Bennett JM (2006). The myelodysplastic syndromes: diagnosis and treatment. Mayo Clin Proc, 81, 104-30.

Suporn C TI, Noppadol S, Wichean M, et al (2011). Myelodysplastic syndrome guideline.

Wallvik J, Stenke L, Bernell P, et al (2002). Serum erythropoietin (EPO) levels correlate with survival and independently predict response to EPO treatment in patients with myelodysplastic syndromes. Eur J Haematol, 68, 180-5.

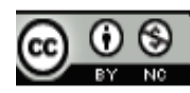

This work is licensed under a Creative Commons AttributionNon Commercial 4.0 International License. 